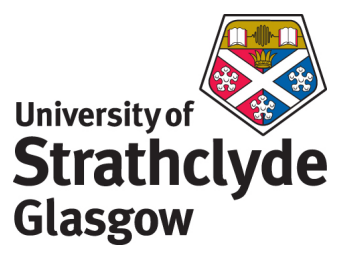

Fletcher, T.M. and Brown, R.E. (2010) Interaction of an Eulerian flue gas plume with wind turbines. In: 29th ASME Wind Energy Symposium, 4-7 January 2010, Orlando, Florida.

http://strathprints.strath.ac.uk/27359/

Strathprints is designed to allow users to access the research output of the University of Strathclyde. Copyright $(C$ and Moral Rights for the papers on this site are retained by the individual authors and/or other copyright owners. You may not engage in further distribution of the material for any profitmaking activities or any commercial gain. You may freely distribute both the url (http://strathprints.strath.ac.uk) and the content of this paper for research or study, educational, or not-for-profit purposes without prior permission or charge. You may freely distribute the url (http://strathprints.strath.ac.uk) of the Strathprints website.

Any correspondence concerning this service should be sent to The Strathprints Administrator: eprints@cis.strath.ac.uk 


\title{
Interaction of an Eulerian Flue Gas Plume with Wind Turbines
}

\author{
Timothy M. Fletcher* and Richard E. Brown ${ }^{\dagger}$ \\ University of Glasgow, Glasgow, G12 8QQ, United Kingdom
}

\begin{abstract}
The reduced availability of sites with the requisite wind resource, planning permission and public acceptance for the placement of wind turbines poses a significant challenge to future expansion of the wind energy industry. Developers increasingly wish to site large turbines in close proximity to industrial plants, but there is uncertainty amongst environmental protection agencies on how best to measure and regulate the impact that wind turbines may have on the dispersion of the gases that are often emitted into the atmosphere from such plants. Several simplified wind turbine-flue stack configurations have been simulated using the Vorticity Transport Model. This model provides a high-fidelity representation of the vortical flow structure within both the wind turbine wake and the plume, and is able to capture the re-direction and dispersion of the plume that occurs due to interaction with the wind turbine. The impingement of the plume on the wind turbine is shown to disrupt the wake structure downwind of the wind turbine, and may induce additional unsteady loading on the turbine rotor. The velocity deficit downwind of the wind turbine influences the rate at which the plume propagates downwind, and results in an increase in the concentration of plume material (which may include pollutant gas and particulates) around the wind turbine. This localized increase in plume concentration is shown to be sensitive to the thrust coefficient at which the wind turbine is operated. The results presented in this paper show that environmental protection agencies are justified in their concerns regarding the placement of wind turbines near to industrial plants, and suggests strongly that the interaction between wind turbines and gas plumes should be investigated further.
\end{abstract}

\section{Nomenclature}

$C_{T} \quad$ rotor thrust coefficient

$g \quad$ gravitational constant

$p \quad$ pressure

$R \quad$ rotor radius

$S \quad$ vorticity source

$S_{b c} \quad$ baroclinic vorticity source

$T$ temperature

$u \quad$ flow velocity

$\begin{array}{ll}u_{b} & \text { flow velocity relative to blade } \\ V_{\infty} & \text { wind speed } \\ \lambda & \text { tip speed ratio, } \Omega R / V_{\infty} \\ \nu & \text { kinematic viscosity } \\ \rho & \text { density } \\ \Omega & \text { rotational speed of the wind turbine } \\ \omega & \text { vorticity } \\ \omega_{b} & \text { bound vorticity }\end{array}$

\section{Introduction}

During the last 20 years the global wind energy industry has grown considerably and many countries now source electricity, to an appreciable extent, from wind turbines. These turbines can be located either in small clusters or in very large wind farms. In smaller countries, a reduction in the availability of sites with the requisite wind resource, planning permission and public acceptance for the placement of wind turbines poses a significant challenge to future expansion of the wind energy industry. Increasingly, wind farm developers wish to place large turbines on sites that are in close proximity to urban or industrialized areas, and even on brownfield sites. In particular, there are an increasing number of cases in which it has been proposed to

\footnotetext{
*Post-doctoral Research Assistant, t.fletcher@eng.gla.ac.uk, Tel: +44 (0)141 3306479 , Fax: +44 (0)141 3305560

${ }^{\dagger}$ Mechan Professor of Engineering, rbrown@eng.gla.ac.uk
} 


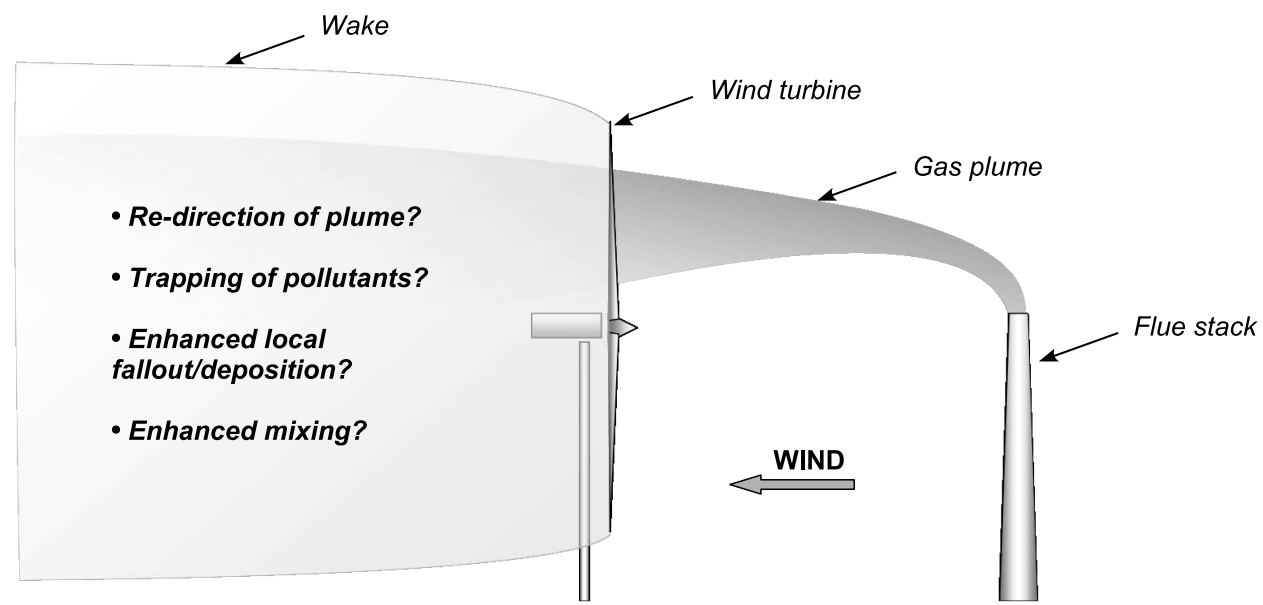

Figure 1. Schematic highlighting some of the potential problems that may arise from the gas plume - wind turbine interaction indicated. Note that the distance between the wind turbine and the flue stack is very much contracted compared to typical practice.

build wind turbines near to industrial plants that emit gases via flue stacks, and environmental protection agencies are unsure how to proceed in specific circumstances where there is concern that the presence of the wind turbines may have an impact on the dispersion of these gases into the surrounding environment.

Figure 1 is a schematic highlighting some of the potential problems that may arise from the interaction between a wind turbine and the gas exhaust from a flue stack. The extraction of power from the wind using turbines results in significant changes in the velocity field surrounding the turbine, and in particular, the formation of a large wake that persists for many rotor diameters downwind. The strong vortices within the wake that is developed by the wind turbine will induce changes in the trajectory of any interacting plume, depending on the portion of the wind turbine rotor that the plume impinges upon. Both the helical structure of the vortices formed downwind of the tips of the turbine blades, and the disordered wake that forms following the breakdown of the tip vortices that invariably occurs several rotor diameters downwind of the turbine, will lead to enhanced mixing of the plume with the surrounding air. The velocity deficit that exists within the wake will lead to the pollutants being concentrated downwind of the turbine, and could possibly enhance locally the fallout and subsequent deposition of particulates that are transported within the plume.

The emission of pollutant gases and their subsequent dispersion within the atmosphere surrounding their source is of concern to, amongst others, public health agencies, urban planning authorities and the developers of industrial plants. As the availability of computers has increased in recent decades, a range of numerical pollutant dispersal models have been developed. These include the ADMS (Atmospheric Dispersion Modeling System) developed in the UK by Cambridge Environmental Research Consultants, and AERMOD, developed jointly by the American Meteorological Society and the US Environmental Protection Agency (see Ref. 1 for an evaluation of both the ADMS and AERMOD approaches). Whilst ADMS and AERMOD have proved to be highly successful when modeling pollutant dispersion, they contain no rigorous capability to model the interaction of airborne pollutants with wind turbines. The objective of this paper is to highlight some of the basic physical characteristics of the interaction between flue gas plumes and wind turbines. The approach to the coupled simulation of both the wind turbine and the plume that is described here has the potential to encapsulate many of the pertinent physical influences on the propagation of gas plumes, and can lend significant insight in those instances where the presence of wind turbines is thought to impact adversely on plume dispersion.

\section{Computational Aerodynamics}

The aerodynamic interaction between a wind turbine and the gas that is exhausted from a flue stack is represented by modeling the mutual interaction of the wake that is developed by the turbine rotor and the vorticity field that is associated with the gas plume. A wind turbine develops a wake in response to the generation of aerodynamic forces on its blades. Concentrated tip vortices and an inboard vortex sheet form 
a helical structure downwind of the blades. This structure persists until its natural instability induces its evolution into a less coherent and significantly more aperiodic field of vorticity. The flue gas plume is also represented as a vortex wake, but one that is created not through the generation of aerodynamic forces, but rather through the evolution of the vorticity field that is created during the emission of a constant mass flux through a finite circular source.

The interaction between a flue gas plume and the wake developed by a horizontal axis wind turbine (HAWT) has been simulated using the Vorticity Transport Model (VTM) developed by Brown, ${ }^{2}$ and extended by Brown and Line. ${ }^{3}$ The VTM enables the high-fidelity simulation of wind turbine aerodynamics and represents particularly well the development of the associated wake structure. After making the physically realistic assumption of incompressibility within the wake, the time-dependent Navier-Stokes equations are cast into the vorticity-velocity form

$$
\frac{\partial}{\partial t} \omega+u \cdot \nabla \omega-\omega \cdot \nabla u=S+\nu \nabla^{2} \omega .
$$

Equation 1 is discretized in finite-volume form on a structured Cartesian mesh. The method is rendered effectively boundary-free as cells may be created, when necessary, on a Cartesian stencil which extends to infinity, using the assumption that there is zero vorticity outside the wake. The velocity field is related to the vorticity field by using a Cartesian fast multipole method to invert the differential form of the Biot-Savart law:

$$
\nabla^{2} u=-\nabla \times \omega .
$$

The vorticity transport equation is solved in inviscid form following the assumption that the Reynolds number of the flow features within the computational domain is sufficiently high. The numerical diffusion and spatial smearing of vorticity within the flow field surrounding the wind turbine is kept at a very low level by using a Riemann problem technique based on the Weighted Average Flux method developed by Toro ${ }^{4}$ to advance equation 1 through time. In practice, the atmosphere in which the wind turbine and the flue gas stack operate is not uniform. Atmospheric flows are subject to wind shear and turbulence, as well as to the effects of temperature gradients. The effects of many of these atmospheric properties on the evolution of flue gas plumes are captured, to some extent, by existing pollutant dispersion models and are therefore not the primary focus of the current paper. Of particular interest here is the influence of the velocity field that is induced by a wind turbine on the trajectory of, dispersion of, and the distribution of material within, a representative flue gas plume.

\section{II.A. Wind Turbine Model}

The aerodynamics of the wind turbine blades is represented using a version of lifting-line theory, in which the vorticity source term $S$ is derived from the temporal and spatial variations of the bound vorticity $\omega_{b}$ on the blades so that

$$
S=-\frac{d}{d t} \omega_{b}+u_{b} \nabla \cdot \omega_{b} .
$$

The forces and moments on the blades are computed using appropriate airfoil performance characteristics that can be modified to account for the effects of the stall delay phenomenon that can occur when the turbine rotor operates at a low tip speed ratio. The rotor that has been simulated in the present study is that tested during Phase VI of the NREL Unsteady Aerodynamics Experiment (UAE). ${ }^{5}$ The rotational speed of the rotor was held constant, whilst the blade pitch was controlled in order to obtain the desired rotor thrust coefficient. The aerodynamic effects associated with the hub assembly and the tower have not been modeled, and the blades are assumed to be rigid. For a detailed description of the blade geometry see Ref. 5; the key properties of the turbine rotor model are summarized in table 1 , however.

Table 1. Rotor data.

\begin{tabular}{lclc}
\hline Type of rotor & rigid & Airfoil & NREL S809 \\
No. of blades & 3 & Rotational speed & $\Omega$ (constant) \\
Rotor radius & $\mathrm{R}$ & Blade pitch & variable
\end{tabular}

3 of 11 


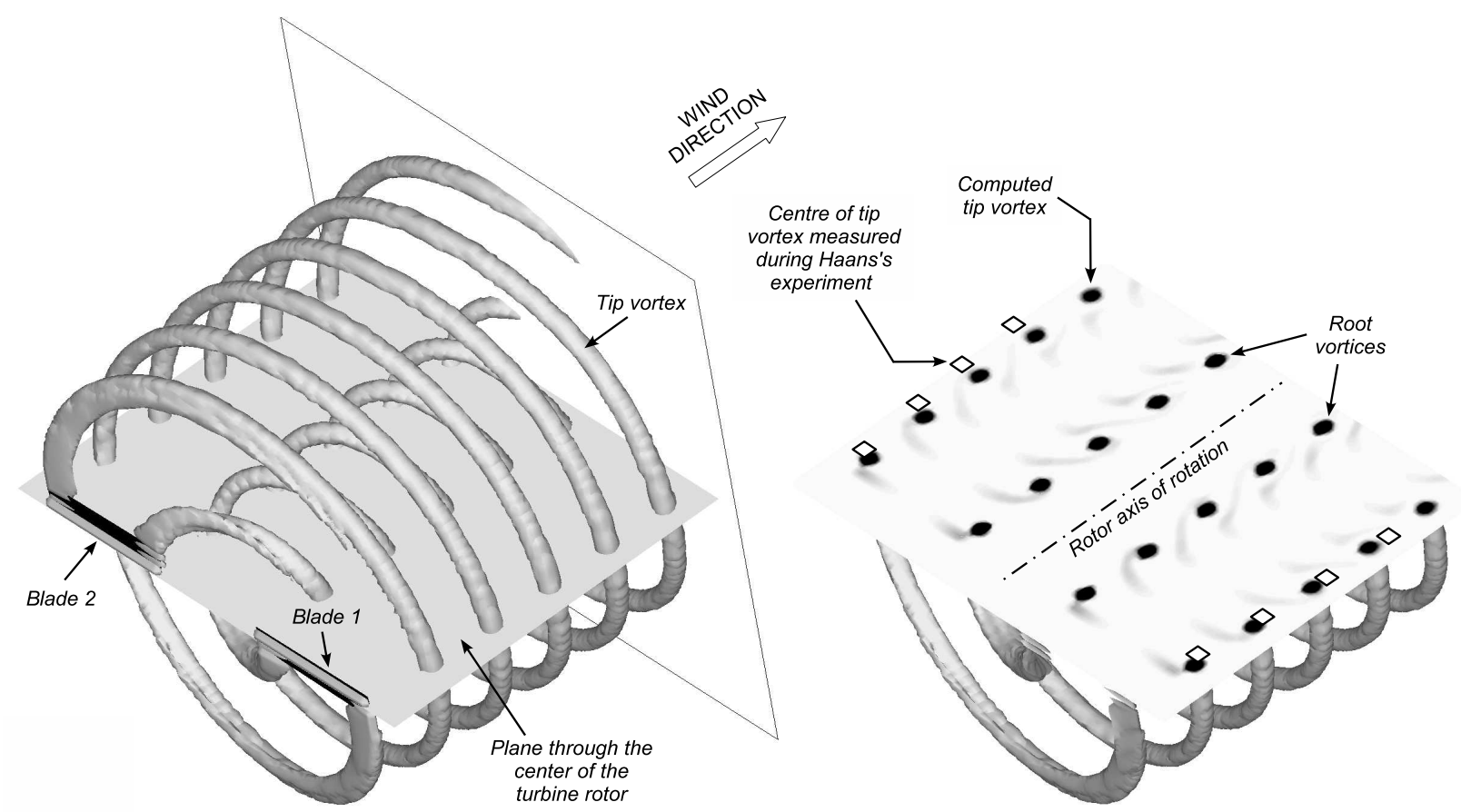

Figure 2. HAWT wake structure, computed using the VTM, compared with data measured during wind tunnel tests of a model rotor by Haans's et al.; $\lambda=8, C_{T}=0.64$. Note: concentrated root vortices are formed as a result of the absence of a hub and hub-blade connection in the VTM simulations.

To represent correctly the distortion of the gas plume by the concentrated vortical structures within the wake developed by the wind turbine, it is essential to predict accurately the formation and evolution of the wake itself. The strength of the vortices that are induced by the wind turbine, and their subsequent evolution, is highly dependent on the distribution of loading on the turbine blades. The VTM has been shown to predict well the distribution of loading on the blades of horizontal axis wind turbines when operating at modest tip speed ratios (see Ref. 6 for comparisons with the NREL Phase VI experimental data when the turbine rotor is operated at a tip speed ratio of 5.4). Figure 2 shows a comparison of the VTM-computed structure of the wake developed by a two-bladed HAWT rotor with corresponding experimental measurements of tip vortex locations made by Haans, Sant, van Kuik and van Bussel. ${ }^{7}$ In order to verify the prediction of wake structure, the rotor was controlled in the VTM simulation such that the thrust coefficient equaled that measured during the wind tunnel experiment $\left(C_{T}=0.64\right)$ when the rotor was operated at a tip speed ratio of eight and a yaw angle of zero. Given the stated error in the experimental measurements and the rather curious variation in the tip vortex positions at opposing azimuthal locations that is evident in the experimental data, figure 2 shows that the VTM is able to predict the trajectories of the tip vortices very well. These results suggest that the VTM is capable of providing a model of the structured wake downwind of the turbine that has a fidelity that is more than sufficient for the simulation of wind turbine - gas plume interaction. In this paper, simulations are described in which the wind turbine was operated at three different thrust coefficients of 0.4 , 0.6 and 0.8 , but where the tip speed ratio was held constant at a value of six.

\section{II.B. Model of the Flue Gas Plume}

The source of the plume of pollutant gas is considered to provide a continuous mass flux, at a fixed location with respect to the wind turbine, and at an elevation some distance above ground level. The gas is emitted normal to a plane containing the axis of rotation of the wind turbine. The source through which the gas is emitted is circular, with a radius of $10 \%$ of the radius of the wind turbine rotor. In all of the simulations, the flue stack was located two rotor diameters upwind of the wind turbine and produced a jet of gas with a mean speed of 1.5 times the local wind speed. It should be noted that the distance between the simulated wind turbine and the flue stack is very small compared to that invariably found in practice. The separation has been chosen at this stage of the investigation, however, to expose the pertinent physics while keeping 


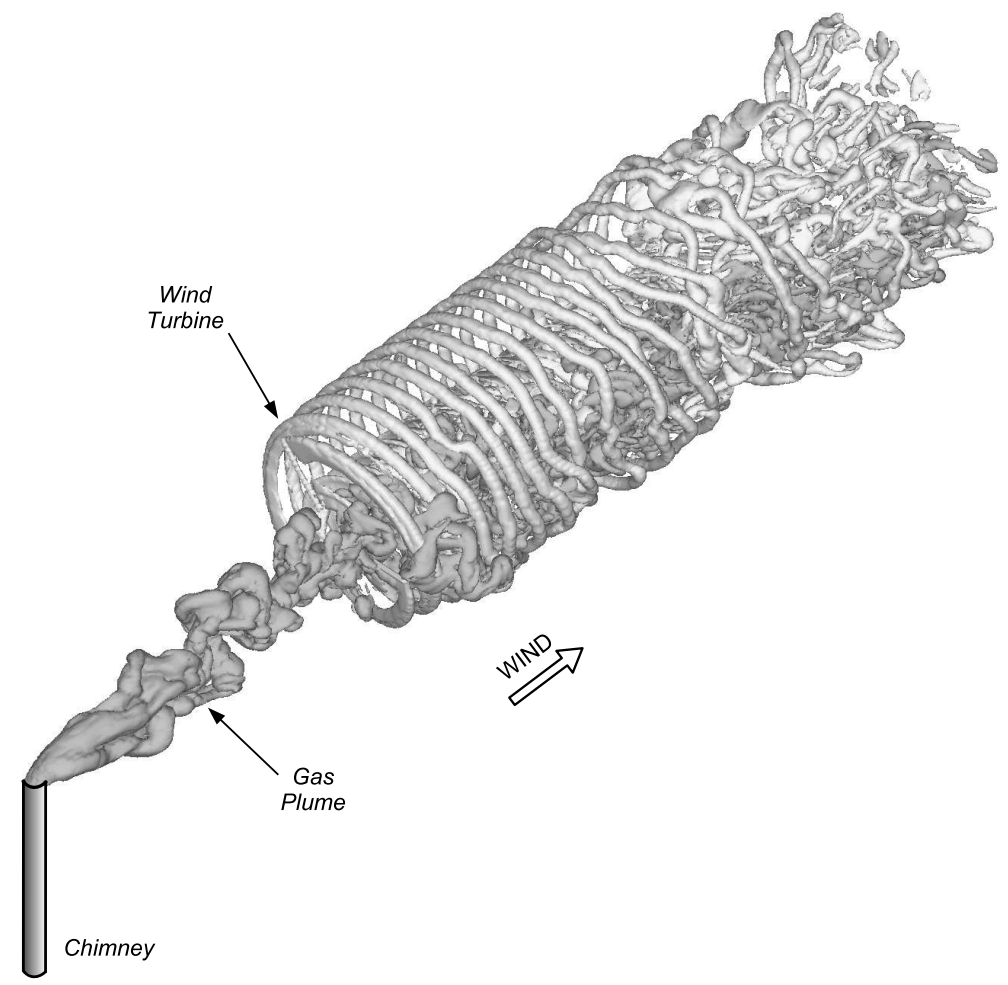

Figure 3. Interaction between the pollutant gas plume exhausted from a flue stack and a wind turbine located downwind; turbine tip speed ratio $=6$.

modest the computational cost of the simulations. Simulations were performed in which the center of the flue was displaced from the axis of rotation of the wind turbine by $0,0.4$ and 0.8 turbine radii in order to assess the significance of the position of the wind turbine rotor relative to the flue stack. Importantly, the gas plumes that are simulated in this paper have neutral buoyancy with the respect to the ambient air.

\section{Implications for Wind Turbine Performance}

The effects of interaction with a wind turbine on the propagation and dispersion of a representative flue gas plume forms the primary focus of the present paper. Notwithstanding this, it is important to recognize the possible implications of such an interaction for the performance of the wind turbine itself. Figure 3 shows a representative instantaneous snapshot of the spatial structure and trajectory of both the gas plume and the wake developed by the wind turbine for the case where the wind turbine is operated at a thrust coefficient of 0.8 and the flue stack exit is displaced $0.4 \mathrm{R}$ outboard from the wind turbine axis. Both the gas plume and the wind turbine wake are rendered using surfaces of constant vorticity magnitude that are chosen to indicate clearly the spatial structure of both the plume and the rotor wake. The figure shows clearly the interaction between the gas plume and the wind turbine.

The swept, but nevertheless tubular, structure of the gas plume as it exhausts from the flue stack does not persist. Rather, after only a very short distance downwind of the stack, the gas plume transforms into a largely disordered structure composed of eddies with a wide range of scales, as shown in figure 3 . The structure of the gas plume demonstrates a close resemblance, at least in a qualitative sense, to the gas plumes that are produced by typical, low-pressure industrial flue stacks. In contrast to the plume, the wake developed by the wind turbine has a distinct structure. The most notable element of this structure is the helical pattern of vortices that is formed downwind of the tips of the turbine blades. Whilst the inherent instabilities within the wake of the turbine do result in its decay to form a disordered structure of finer-scale vortices after the wake has convected many rotor diameters downwind, it is clear from figure 3 that the morphology of wind turbine - gas plume interaction is one of a largely disordered plume impinging on a turbine, and interacting with the highly structured wake that the turbine produces downwind of itself. 


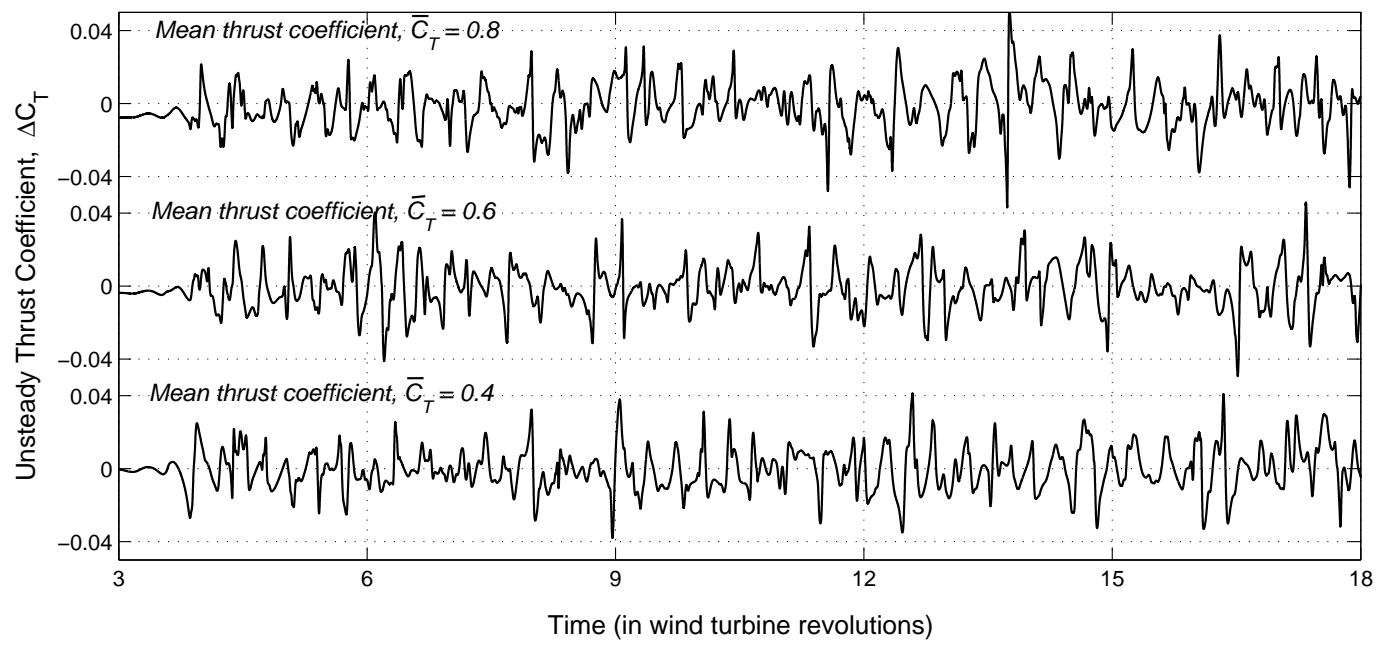

Figure 4. Unsteady performance of the wind turbine as a result of interaction with the gas plume whilst operating at three different mean thrust coefficients (note the offsets applied to the thrust axis).

Figure 4 shows the unsteady component of the thrust coefficient produced by the turbine, $\Delta C_{T}=C_{T^{-}} \bar{C}_{T}$, where $\bar{C}_{T}$ is the mean thrust coefficient. In figure $4, \Delta C_{T}$ is shown for the configurations where the centerline of the flue stack is displaced $0.4 \mathrm{R}$ from the turbine axis, but for three different cases with $\bar{C}_{T}=0.4,0.6$ and 0.8. Figure 4 demonstrates that the thrust coefficient at which the wind turbine rotor operates, in all of the operating conditions that are represented, exhibits significant unsteadiness. Increasing the mean thrust coefficient of the wind turbine rotor (by means of greater blade pitch) has the effect of increasing the amplitude of the fluctuations in the thrust that is developed by the turbine, as shown in figure 4. Interestingly, however, the amplitude of the fluctuations are approximately proportional to the mean thrust coefficient of the rotor, and therefore amount to a relatively consistent proportion of the overall thrust coefficient that is produced by the turbine.

The data presented in figure 4 is intended to provide insight into the unsteadiness in the aerodynamic performance of the rotor that might occur when the wind turbine is impinged upon by a gas plume. The impulsive fluctuations in the loading on the turbine that are shown in figure 4 are created when the turbine blades sweep through the vortical structures or eddies that are contained within the gas plume. These eddies are responsible for inducing strong localized fluctuations in the inflow velocity that is experienced by parts of the turbine blades. The distinct absence of periodicity in the rotor loading that is shown in figure 4 is because the plume is inherently turbulent (and hence random) in nature. The natural instabilities within wakes lead to a progressive breakdown of their structure over time, such that the magnitude of the associated fluctuations in the velocity field diminish with distance from their source. In addition, the turbulence that is ordinarily present within the atmosphere would accelerate the decay of the plume into finer scale eddies. Indeed, the close proximity of the flue stack to the wind turbine and the absence of atmospheric turbulence within the simulations implies that the magnitude of the fluctuations in rotor thrust coefficient that are exhibited here are larger than those that would be experienced in most practical systems. It should be noted, however, that even low levels of additional forcing are likely to compound the fatigue of the turbine that is caused by other sources of unsteadiness in the rotor inflow, and to contribute to reducing the operational life of the turbine.

\section{Plume - Wind Turbine Interaction}

Figure 5 demonstrates the mutual distortion of the modeled gas plume and the wind turbine wake for a configuration where the centerline of the flue stack is displaced $0.8 \mathrm{R}$ outboard from the axis of rotation of the wind turbine. Both figures $5(\mathrm{a})$ and $5(\mathrm{~b})$ are instantaneous snapshots of the combined vorticity field that is generated within the wind turbine wake and the gas plume. Figure $5(\mathrm{a})$ shows contours of vorticity magnitude on a horizontal plane that includes the axis of rotation of the wind turbine, whilst figure 5(b) 


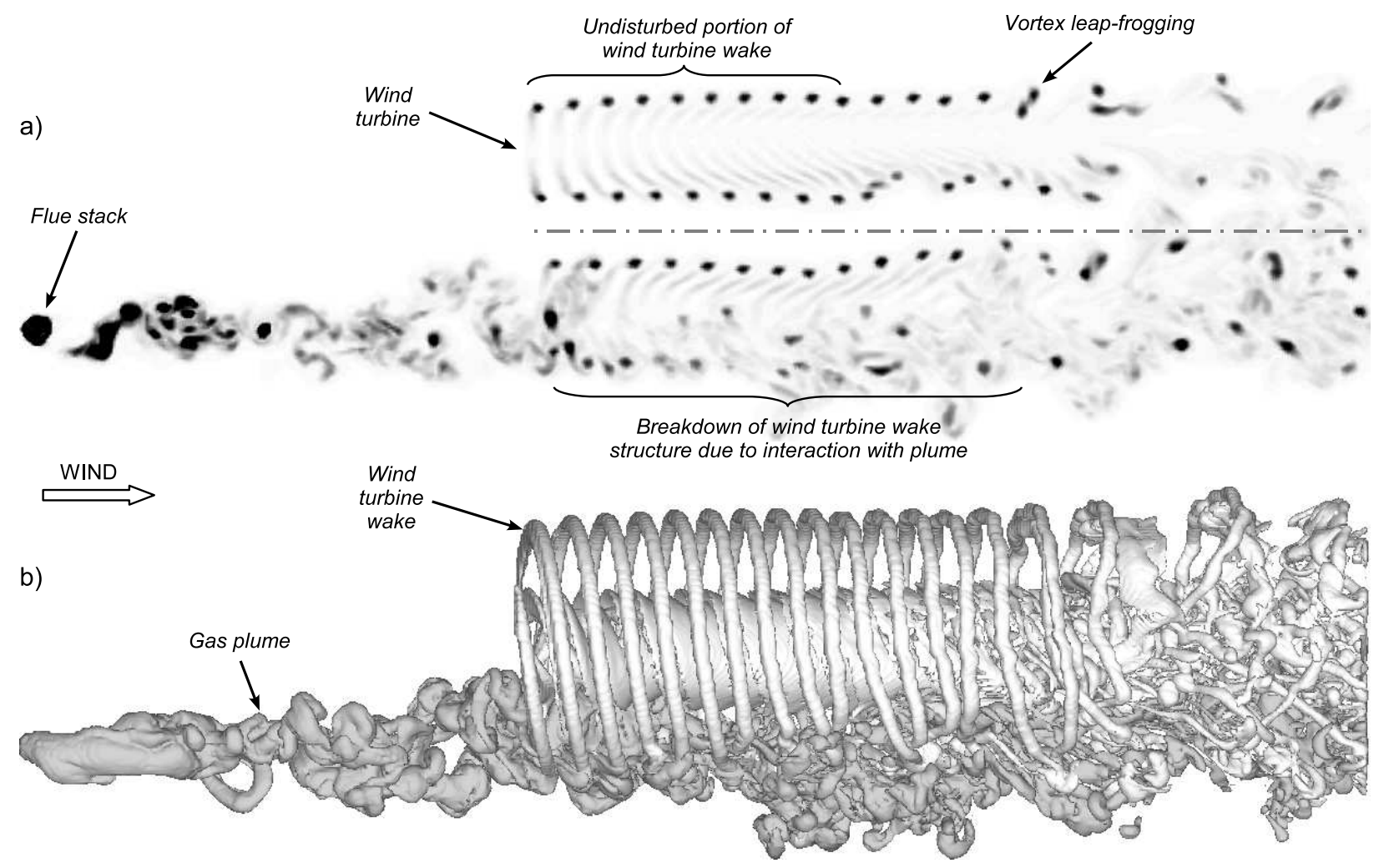

Figure 5. Effect on wind turbine wake development of interaction with the gas plume produced by a flue stack displaced by $0.8 \mathrm{R}$ from the axis of the wind turbine; a) contours of vorticity magnitude on a horizontal plane that includes the wind turbine axis of rotation; b) the plume (dark) and the wind turbine wake (light) rendered as three-dimensional surfaces of constant vorticity magnitude.

shows the plume (dark) and wind turbine wake (light) rendered as three-dimensional surfaces on which the vorticity has constant magnitude. Figures $5(\mathrm{a})$ and 5(b) demonstrate a clear lateral asymmetry in the vorticity field that is produced by the turbine as its wake develops downstream. The tip vortices formed downwind of the blades, when on the opposite side of the turbine to the flue stack, form a helical structure which persists until 2-3 rotor diameters downwind of the turbine. Further downstream, the onset of the wake breakdown that is triggered by the natural instabilities within the wake leads to a rapid disordering of the wake structure. Figure 5(a) demonstrates, however, that the formation and evolution of the turbine wake downwind of the part the rotor that is directly impinged upon by the plume is disrupted significantly. Despite the plume impinging on only a relatively small portion of the rotor disk, the helical pattern of the tip vortices is disrupted almost immediately downstream of the rotor as a result of interaction with the vorticity within the plume.

Within limits, the distribution of vorticity magnitude within the plume can be treated as an analogue for the concentration of material that is ejected from the plume into the flow. Figure 6 indicates the spatial distribution of ejected material along the length of the isolated plume. This distribution is obtained by integrating the total quantity of ejected material that is present on a series of slices that are oriented perpendicular to the wind direction. In figure 6 , the concentration of material along the length of the plume is scaled using the material concentration at the top of the flue. Figure 6 demonstrates that a large concentration of material exists near to the flue stack, but that the concentration reduces sharply such that, at a distance of approximately $4-5$ turbine diameters downwind of the flue stack, the concentration of material within the plume is relatively uniform. The high concentration of material near to the flue stack is caused by the largely vertical trajectory of the plume as it is exhausted from the flue. The sharp reduction in the concentration of plume material with distance downwind is thought to occur, in part, as the rate of propagation of the plume in the vertical direction diminishes and the plume is transported downstream by the wind. It should be noted that the distribution of material within the plume near to the flue stack is 


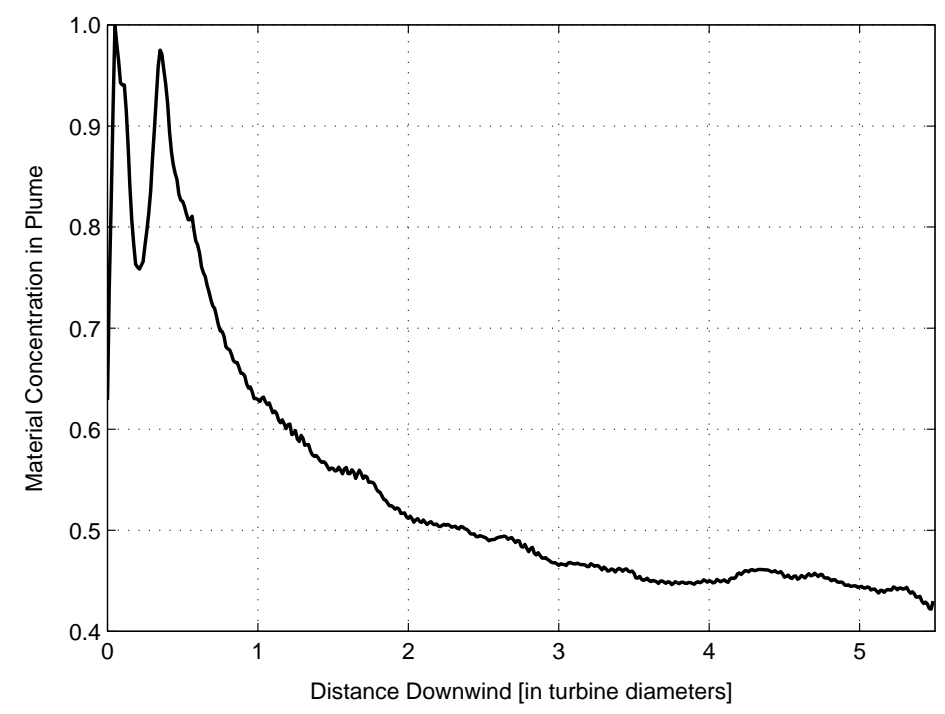

Figure 6. Distribution of ejected material along the length of the isolated plume, obtained by integrating the total quantity of ejected material on slices perpendicular to the wind direction.

strongly governed by the geometry of the flue exit and by the details of the method by which the jet of pollutant gas is modeled.

Wind turbines produce electrical power by extracting kinetic energy from the wind, but, in so doing, create a velocity deficit within the flow downwind of the turbine. The extent to which the air is decelerated as it passes through the turbine is related to the loading on the turbine rotor, and thus varies with the thrust coefficient at which the rotor is operated. If the gas plume produced by a flue propagates through an operational wind turbine, then the rate at which the plume subsequently convects downwind will be reduced by the lower local flow speed downwind of the turbine. Figure 7 shows the effect of the wind turbine on the distribution of material along the length of the plume. In this figure, the concentration of material at each windward location within the plume is scaled using the concentration within the isolated plume (as was presented in figure 6). A relative material concentration of greater than one therefore indicates that the plume contains more material at a particular windward location than the isolated plume and vice versa. Figure 7 (a) shows the effect on the plume material distribution of changing the thrust coefficient at which the wind turbine is operated. In this case the relative positions of the flue stack and the turbine are kept constant. Figure 7(b) shows the sensitivity of the distribution of plume material to the lateral displacement of the flue stack with respect to the axis of rotation of the wind turbine. In this case the rotor thrust coefficient was held constant at 0.8. In all cases the turbine was located two rotor diameters downwind of the flue.

Figure 7(a) demonstrates a fairly strong correlation between the thrust coefficient of the wind turbine and the distribution of material within the plume. A distinct increase in material concentration is observed close to the wind turbine. This increase in material concentration around the wind turbine is highest when the turbine rotor operates at high thrust coefficient, and is caused by the reduction in the flow speed that is experienced by the plume as it passes through the wind turbine rotor. When the thrust coefficient of the wind turbine rotor is lower, the sensitivity of the material concentration within the plume to the loading on the turbine rotor is considerably less pronounced. Figure 7(a) shows that the material concentration within the plume, downwind of the wind turbine rotor, decreases below that within the isolated plume at equivalent windward locations. This distortion of the distribution of material within the plume downwind of the turbine is fundamentally a result of the conservation of mass in the plume and the recovery of the flow velocity within the wind turbine wake to the ambient wind velocity. The reduction in material concentration with distance downwind of the turbine is strongly dependent on the thrust coefficient of the wind turbine rotor. Figure 7 (a) shows that, in general, the material concentration within the plume at significant distance downstream of the turbine is higher when interacting with a more lightly loaded turbine rotor. This observation suggests that the change in the local flow speed across the wind turbine governs the expansion and contraction of the 


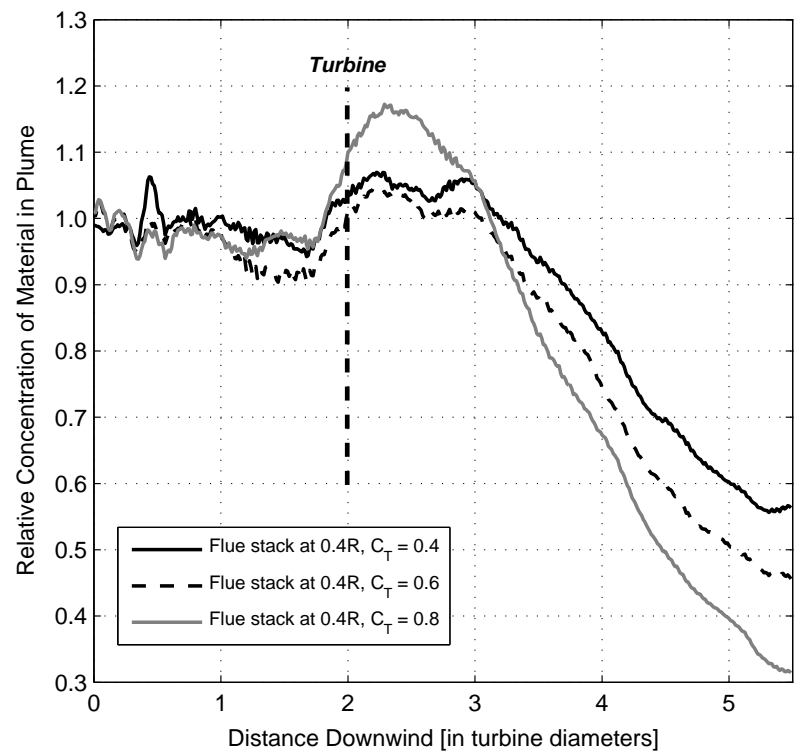

a) Effect of rotor $C_{T}$ (flue-turbine geometry constant).

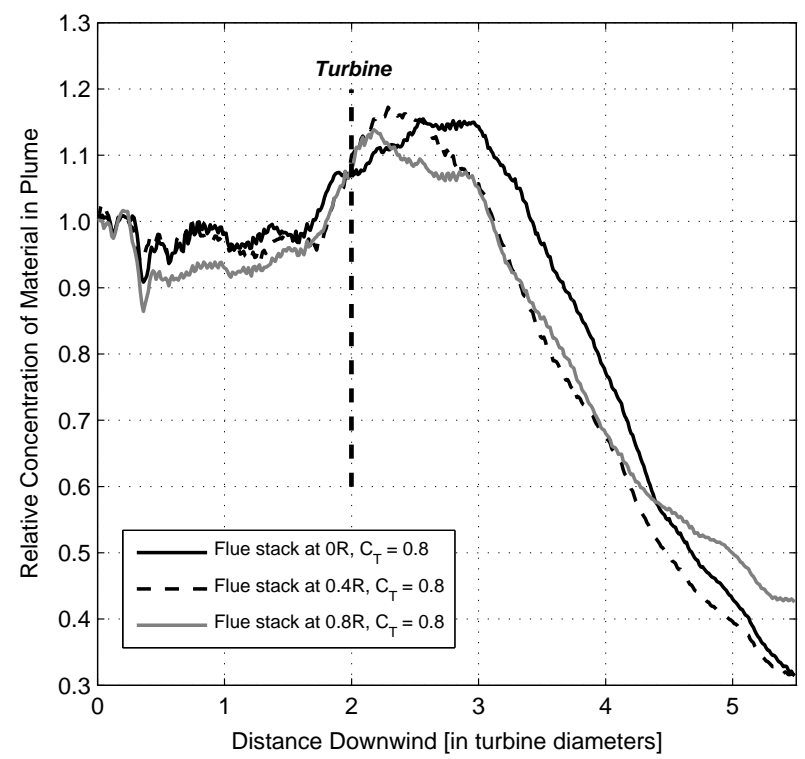

b) Effect of flue-turbine geometry (rotor $C_{T}$ constant).

Figure 7. Effect of the wind turbine on the distribution of material along the length of the plume. The concentration of material at each windward location within the plume is scaled using the concentration within the isolated plume.

plume, and therefore the concentration of material downwind of the turbine. Figure 7(b) indicates, to some extent, that if the flue stack is co-located along the rotor axis, then the material concentration within the plume downwind of the turbine is higher than if the flue stack is located further outboard. This trend does not persist beyond 4-5 rotor diameters downwind of the turbine, however, and figure $7(\mathrm{~b})$ suggests that the sensitivity of the material concentration within the plume to the location of the flue stack relative to the turbine is strongly dependent on the detailed characteristics of the interaction between the plume and the rotor wake downwind of the turbine.

The plume that is formed at the exit of an industrial flue stack often contains a significant concentration of suspended particulate matter. Perhaps the most common species of particulate in practice is soot, formed from the incomplete combustion of hydrocarbons. Whilst the simulation of particle transport is a complex multiphase problem that must be modeled with sufficient physical realism, the results presented in figure 7 suggest that the deposition of particulates on to the ground near to the wind turbine could be enhanced by the interaction between the plume and the turbine rotor. The link between plume-wind turbine interaction and the enhanced concentration of contaminents in the ground near to the sites of wind turbines therefore warrants further study. The problem might be further exacerbated by the fact that wind turbines are most commonly clustered together into so-called wind farms, whereby the impact that each individual wind turbine has on the propagation of the plume could combine cumulatively, to some extent, to result in an enhancement of the deposition of particulate matter on the ground in and around the wind farm.

\section{Plume Buoyancy}

Whilst the aerodynamic model used in the present study represents well the characteristic eddies within the gas plume, and preserves the inherent vorticity as the plume evolves in time and space, there are nevertheless several key physical effects that have thus far been omitted. In the present study, the temperatures of the plume and the surrounding air within the atmosphere were assumed to be equal. In reality, however, the temperature (or chemical composition) of the ejected material may be different to that of the ambient air. Any absence of thermal equilibrium between the plume and the surrounding air manifests as a buoyancy force on the plume that will cause it to rise or fall vertically until its temperature reaches equilibrium with the surrounding air. This process is closely related to the concept of atmospheric stability, whereby a column of air warmed by high surface temperatures will tend to rise through the surrounding (lower temperature) air within the atmosphere. Buoyancy has been neglected in the present study because its primary effect 


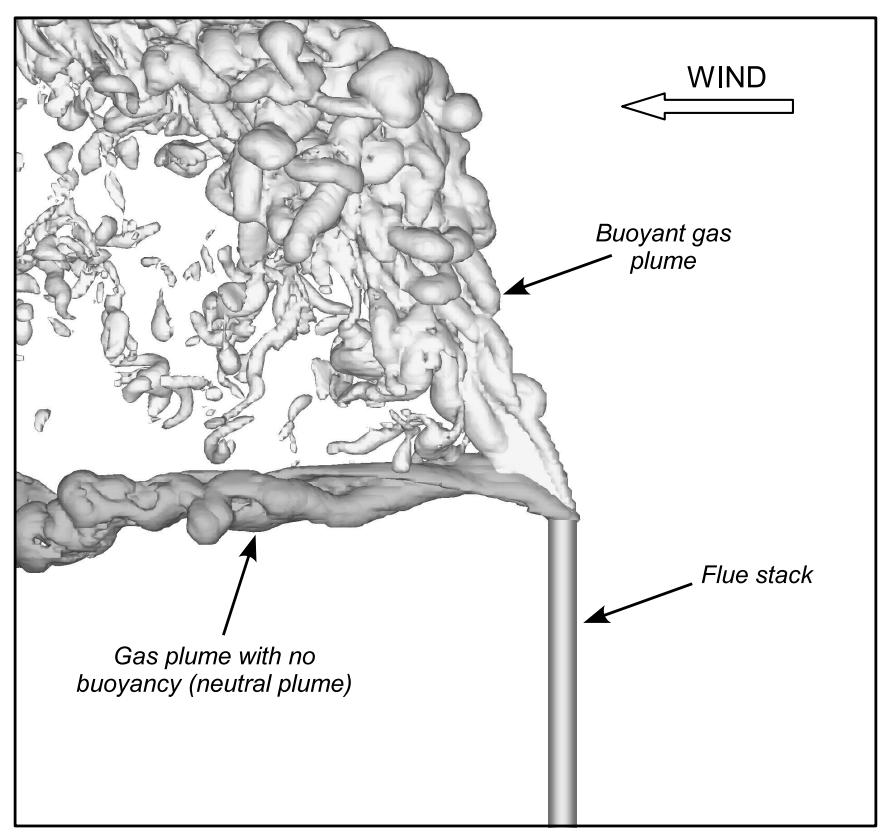

Figure 8. Effect of buoyancy on the trajectory of the plume.

is to alter the trajectory of the plume after it is exhausted from the flue. Buoyancy does not influence fundamentally the physics of the interaction between the vorticity fields that are associated with the plume and the wake that is developed by the wind turbine.

The basic effects of a non-uniform temperature distribution within the flow field that is caused by a warm plume passing through cooler surrounding air can be incorporated within the Vorticity Transport Model by evolving alongside the vorticity field a scalar temperature field, and deducing the associated baroclinic vorticity source within the vorticity transport equation from the associated temperature gradients. The baroclinic source of vorticity $S_{b c}$, stated in terms of pressure $p$, and density $\rho$, is

$$
S_{b c}=\frac{1}{\rho^{2}}(\nabla \rho \times \nabla p) .
$$

If the ideal-gas equation of state $p=\rho R T$ can be assumed to characterize adequately the thermodynamics of the plume (where $T$ is temperature and $R$ is the specific gas constant of the fluid), then the baroclinic vorticity source can be expressed in terms of the gradients of the temperature and pressure fields, so that

$$
S_{b c}=-\frac{1}{\rho T}(\nabla T \times \nabla p) .
$$

If the topography surrounding the flue stack is assumed to be relatively smooth, such that the pressure at any given altitude is essentially constant over appreciable horizontal length scales, then the pressure gradient can be approximated as being non-zero in only the vertical direction. By applying the hydrostatic pressure relationship $\nabla p=[0,0,-\rho g]$ the baroclinic vorticity source within equation 5 can be computed explicitly from the temperature field.

Figure 8 shows the effect of buoyancy on the simulated trajectory of a plume. The vertical pressure gradient applied to equation 5 creates additional vorticity within the buoyant plume that is proportional to the horizontal temperature gradients within the flow. It should be noted that the influence of buoyancy forces on the dynamics of the plume was purposely exaggerated in this case to demonstrate clearly the sensitivity of the trajectory of the plume to the baroclinic source of vorticity. Figure 8 shows that the baroclinic vorticity source results in the buoyant plume rising to greater altitude over a shorter ground distance than the neutral plume. Whilst figure 8 demonstrates the effect of temperature gradients within the flow, buoyancy effects can also occur when the chemical composition is such that the gas that is exhausted from the flue stack has 
a different molecular weight to that of the surrounding air. A gas plume that has a higher molecular weight, and is therefore more dense than the surrounding air, will tend to sink whereas a gas with a lower molecular weight will tend to rise. To encapsulate faithfully the effects of buoyancy within the simulations, therefore, the variations in both the temperature and molecular weight of the plume with respect to the ambient air must be modeled.

\section{Conclusion}

The accurate numerical simulation of the interaction between wind turbines and flue gas plumes requires the vortical flow structure within both the turbine wake and the plume to be modeled with a high degree of realism. The Vorticity Transport Model enables the interaction between the turbine wake and the plume to be simulated from first principles and, through the analysis of several simplified wind turbine-flue stack configurations, has allowed some of the generic effects of the wind turbine-plume interaction to be exposed. The impingement of the plume on the wind turbine is shown to disrupt the wake structure downwind of the wind turbine, and may induce additional unsteady loading on the turbine rotor. The velocity deficit downwind of the wind turbine influences the rate at which the plume propagates downwind, and results in an increase in the concentration of plume material (which may include pollutant gas and particulates) around the wind turbine. This localized increase in plume concentration is sensitive to the thrust coefficient at which the wind turbine is operated. Although the effects of particulate transport and fallout were not modeled, the results presented here suggest that the deposition of particulates onto the ground near to the wind turbine could be enhanced by the interaction between the plume and the turbine rotor. The results presented in this paper show that environmental protection agencies are justified in their concerns regarding the placement of wind turbines near to industrial plants, and strongly suggests that the interaction between wind turbines and gas plumes should be investigated further in order to quantify clearly the risks associated with future strategies regarding the use of land near to industrial sites.

\section{References}

\footnotetext{
${ }^{1}$ Hanna, S.R., Egan, B.A., Purdum, J., and Wagler, J., "Evaluation of the ADMS, AERMOD, and ISC3 Dispersion Models with the OPTEX, Duke Forest, Kincaid, Indianapolis and Lovett Field Datasets," International Journal of Environment and Pollution, Vol. 16, No. 1-6, 2001, pp. 301-314.

${ }^{2}$ Brown, R.E., "Rotor Wake Modeling for Flight Dynamic Simulation of Helicopters," AIAA Journal, Vol. 38, No. 1, 2000, pp. 57-63.

${ }^{3}$ Brown, R.E., and Line, A.J., "Efficient High-Resolution Wake Modeling using the Vorticity Transport Equation," AIAA Journal, Vol. 43, No. 7, 2005, pp. 1434-1443.

${ }^{4}$ Toro, E.F, "A Weighted Average Flux Method for Hyperbolic Conservation Laws," Proceedings of the Royal Society of London, Series A: Mathematical and Physical Sciences, Vol. 423, No. 1865, 1989, pp. 401-418.

${ }^{5}$ Hand, M.M., Simms, D.A., Fingersh, L.J., Jager, D.W., Cotrell, J.R., Schreck, S., and Larwood, S.M., "Unsteady Aerodynamics Experiment Phase VI: Wind Tunnel Test Configurations and Available Data Campaigns," NREL Report TP-500-29955, 2001.

${ }^{6}$ Fletcher, T.M., and Brown, R.E., "Simulating Wind Turbine Interactions using the Vorticity Transport Model," 28th ASME Wind Energy Symposium, World Center Marriott, Orlando, Florida, USA, 5-8 January 2009.

${ }^{7}$ Haans, W., Sant, T., van Kuik, G., and van Bussel, G., "Measurements of Tip Vortex Paths in the Wake of a HAWT Under Yawed Flow Conditions," Journal of Solar Energy Engineeering, Vol. 127, No. 4, 2005, pp. 456-463.
} 